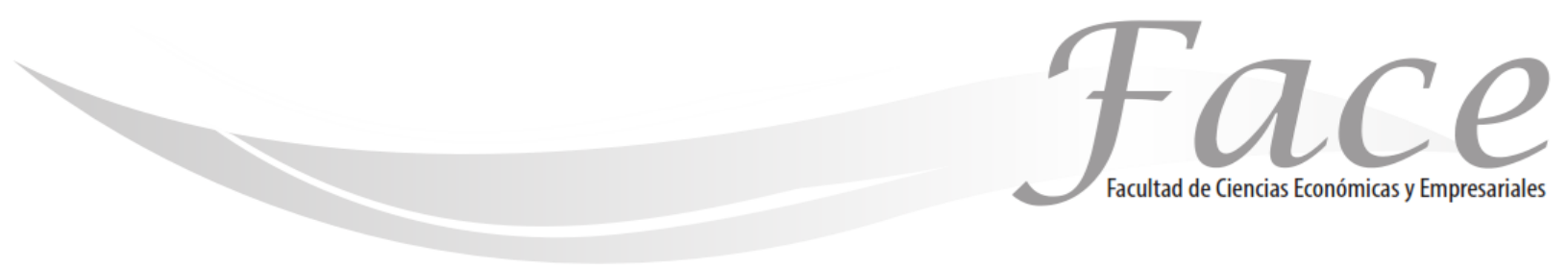

ISSN Impreso: 1794-9920 ISSN Electrónico: 2500-9338

Volumen $18-\mathrm{N}^{\circ} 2$

Año 2018

Págs. $67-77$

\title{
ANÁLISIS DE CUMPLIMIENTO DEL IMPUESTO AL CONSUMO EN LOS RESTAURANTES DEL MUNICIPIO DE FLORENCIA
}

\author{
Maria Yenny Fajardo * \\ Enlace ORCID: https://orcid.org/0000-0002-3846-149X \\ Guineth Facundo Vargas \\ Enlace ORCID: https://orcid.org/0000-0002-8956-5862 \\ Carlos Alberto Gómez Cano *** \\ Enlace ORCID: https://orcid.org/0000-0003-0425-7201
}

Fecha de Recepción: 15 de junio 2018

Fecha de Aprobación: 7 de Octubre 2018

\section{Resumen:}

El estudio abordado hace referencia al análisis de la aplicación del nuevo impuesto al Consumo aprobado en la modificación del Estatuto Tributario establecido en la Ley 1607 de 2012, el análisis abarca a los propietarios de 104 empresas de servicio de restaurante del Municipio de Florencia Caquetá, periodo 2013-2014. Se aplicó encuesta cuyo análisis estadístico se hizo a través del paquete SPSS, el cual reviste todos los aspectos elementales para la preparación de gráficos y estadísticas descriptivas. Los resultados arrojados muestran que de Las empresas encuestadas y que pertenecen al el Régimen Común, el $78 \%$ de los establecimientos se encuentran al día con la presentación de las declaraciones bimestrales del impuesto al consumo y el $22 \%$ restante, no contestaron, lo que nos permite establecer que existe aún desconocimiento de la norma y han tenido dificultad en conocer la norma para dar aplicabilidad con esta nueva obligación tributaria.

Palabras clave: Impuesto al consumo, obligación tributaria, prestador de servicio de restaurante.

\footnotetext{
*Docente Universiad de la Amazonia - , Magister Universidad De Manizales en Desarrollo sostenible y medio Ambiente - Umanizales-Colombia. Correo: mfajard084@hotmail.com

** Docente Universiad de la Amazonia - , Magister Universidad De Manizales en Desarrollo sostenible y medio Ambiente - Umanizales-Colombia. Correo: g.facundo@udla.edu.co

*** Contador Público, Administrador Público, Especialista en Gestión Pública de la Escuela Superior de Administración Pública - ESAP -. Contacto: carlosgomez325@gmail.com
} 


\title{
EVALUATION OF CUSTOMER SATISFACTION IN THE FINANCIAL SECTOR OF THE MUNICIPALITY OF PAMPLONA - COLOMBIA.
}

\begin{abstract}
:
The study addressed refers to the analysis of the application of the new Consumption tax approved in the modification of the Tax Statute established in Law 1607 of 2012, the analysis covers the owners of 104 restaurant service companies of the Municipality of Florencia Caquetá, period 2013-2014. A survey was applied whose statistical analysis was made through the SPSS package, which covers all the elementary aspects for the preparation of graphs and descriptive statistics. The results show that of the surveyed companies that belong to the Common Regime, $78 \%$ of the establishments are up to date with the presentation of the bi-monthly statements of the consumption tax and the remaining $22 \%$ did not answer, which allows to establish that there is still ignorance of the rule and have had difficulty knowing the rule to give applicability with this new tax obligation.
\end{abstract}

Keywords: Tax on consumption, tax liability, restaurant service provider.

\section{AVALIAÇÃO DA SATISFAÇÃO DO CLIENTE NO SETOR FINANCEIRO DO MUNICÍPIO DE PAMPLONA - COLÔMBIA}

Resumo:

O estudo abordado refere-se à análise da aplicação do novo Imposto de Consumo aprovado na modificação do Estatuto Fiscal estabelecido na Lei 1607 de 2012, a análise abrange os proprietários de 104 empresas de serviços de restauração do Município de Florencia Caquetá, período 2013- 2014 Foi aplicado um survey cuja análise estatística foi feita através do pacote SPSS, que abrange todos os aspectos elementares para a elaboração de gráficos e estatística descritiva. Os resultados mostram que das empresas pesquisadas que pertencem ao Regime Comum, $78 \%$ dos estabelecimentos estão em dia com a apresentação das declarações bimensais da taxa de consumo e os $22 \%$ restantes não responderam, o que permite estabelecer que ainda há desconhecimento da regra e dificuldade em conhecer a regra para dar aplicabilidade com essa nova obrigação tributária.

Palavras-chave: Setor Financeiro, Modelo Servqual, Qualidade de serviço. Setor Financeiro, Modelo Servqual, Qualidade de serviço, estatística. 


\section{INTRODUCCIÓN:}

Considerando que mediante Ley 1607 del 26 de Diciembre de 2012 en sus artículos 71 al 83, se adicionó en el Estatuto Tributario Colombiano el Impuesto Nacional al Consumo (INC), mediante los artículos 512-1 al 512-13, que entraría en vigencia a partir del 1 de enero de 2013 en todo el territorio nacional, con excepción de los departamentos de Amazonas y el archipiélago de San Andrés, Providencia y Santa Catalina; y luego con la Ley 1819 del 29 de diciembre del 2016 mediante los artículos207 al 213 adicionó los artículos 512-15 al 512-21 agregando al INC dos nuevos hechos generadores al INC.

Este impuesto grava únicamente las siguientes actividades: La prestación del servicio de telefonía móvil al consumidor final, con tarifa del $4 \%$, la venta o importación de la mayoría de vehículos, botes y aerodinos a favor de un consumidor final siempre y cuando no sean un activo fijo para el vendedor, con tarifas del $8 \%$ y $16 \%$ según el tipo de vehículo, bote 0 aerodino los servicios de restaurantes, bares, tabernas y discotecas, con una tarifa del $8 \%$ dar bolsas plásticas para cargar o llevar productos enajenados por los entes comerciales por valor de $\$ 20$ a partir del 1 de julio del 2017 con incremento anual de $\$ 10$ hasta el año 2020; por otro lado por la venta de productos transformados de la planta cannabis psicoactivo o no psicoactivo asignándose el 16\% de impuesto.

En este sentido la reforma tributaria del 2012 en cuanto a la actividad de los servicios de restaurantes, bares, tabernas y discotecas, incluyó en la categoría de restaurante a las cafeterías, autoservicios, panaderías, fruterías heladerías y pastelerías, así como todo bar, taberna o discoteca, que sean sitios en donde se preparen comidas y/o bebidas alcohólicas para el consumo por parte del consumidor final en el sitio de la venta 0 para ser llevadas o enviadas a domicilio, y que sean negocios que no funcionen bajo franquicia, ni se dediquen al servicio de catering empresarial, ni se trate de restaurantes que funcionen dentro de las instituciones educativas reconocidas por el estado (Art. 476 del E.T.) sin importar si son negocios de personas naturales 0 jurídicas, quedaron exonerados de la responsabilidad del impuesto a las ventas -IVA, pero tendría que responder con el INC, con una tasa del $8 \%$.

Por otro lado la Reforma Tributaria para el impuesto nacional al consumo, determinó dos clasificaciones de los obligados en declarar este impuesto; el régimen común y el régimen simplificado. En este sentido se incorporó un nuevo artículo que estipula que al régimen simplificado del impuesto nacional al consumo de restaurantes y bares al que hace referencia en el Estatuto Tributario, pertenecen las personas naturales y jurídicas que en el año anterior hayan obtenido ingresos brutos totales provenientes de la actividad inferiores a cuatro mil (4.000) UVT. Luego con Reforma Tributaria del 2014, ya no podrían seguir perteneciendo a este régimen las personas jurídicas, por lo que solamente pertenecen a este régimen las personas naturales; por otro lado en el año en la reforma tributaria en el 2016 nuevamente se modifica este articulado disminuyendo el tope de ingresos obtenidos en el año inmediatamente anterior y agregando una nueva condición para poder pertenecer a este régimen; de esta manera la modificación establece que al régimen simplificado del impuesto nacional al consumo de restaurantes y bares a que hace referencia el numeral 3 del artículo 512-1 de este Estatuto, pertenecen las personas naturales que cumplan la totalidad de las siguientes condiciones: Que en el año anterior hayan obtenido ingresos brutos totales, provenientes de la actividad, inferiores a tres mil quinientas (3.500) UVT. y que tengan máximo un establecimiento de comercio, sede, local o negocio donde ejercen su actividad.

Por lo tanto se consideró importante abordar este tema para verificar el cumplimiento de los comerciantes del régimen simplificado responsables del impuesto nacional al consumo, entre ellos, la obligación de presentar anualmente la declaración simplificada por sus ingresos relacionados con la prestación del servicio de restaurantes, bares y similares, como lo estableció el Decreto reglamentario 2623 de 2014. Esta declaración sería simplemente informativa, pero si no se presenta oportunamente implicará para los responsables sanciones de extemporaneidad que corresponderá al $5 \%$ del total de los ingresos de las prestaciones de servicios que se hacen durante el año anterior y se multiplicará por cada mes 0 fracción de mes en que se lleguen a demorar en presentarla, tal como lo establece el Estatuto Tributario en su artículo 641.

De esta forma el artículo 1.3.3.7. del Decreto Único Tributario 803 del 24 de abril del 2014, en su artículo 6, contiene las responsabilidades formales que las personas naturales responsables del régimen simplificado del impuesto nacional al consumo deben cumplir, entre las que se contempla obligaciones y prohibiciones; y dentro de las obligaciones está la de presentar la declaración simplificada de ingresos en forma anual.

Esta investigación se realizó para responder la siguiente pregunta problema: ¿Qué incidencia tiene para los 
prestadores de servicio de restaurantes del municipio de Florencia responsables del régimen simplificado del impuesto nacional al consumo el no cumplimiento de sus obligaciones tributarias?. El desarrollo de la investigaciòn logró establecer la caracterización de las empresas prestadoras de servicio de restaurante de la Ciudad de Florencia, de cuyo análisis se pudo establecer el cumplimiento o no de las obligaciones tributarias frente al impuesto nacional al consumo; dicho resultado se resuelve con la metodología desarrollada en el proyecto y que se describe a continuación.

\section{METODOLOGÍA:}

La investigación es de carácter descriptivo por cuanto expone situaciones y eventos. Esto es, decir cómo es y se manifiesta determinado fenómeno. Los estudios descriptivos buscan especificar las propiedades importantes de personas, grupos, comunidades o cualquier otro fenómeno que sea sometido a análisis. Tal como lo establece Dankhe, 1986. Miden y evalúan diversos aspectos, dimensiones o componentes del fenómeno 0 fenómenos a investigar. Asi mismo es de carácter explicativo, según Hernandez \& Fernandez, Baptista (1997), porque está dirigida a responder a las causas de los eventos físicos o sociales. Como su nombre lo indica, su interés se centra en explicar por qué ocurre un fenómeno y en qué condiciones se da éste, o por qué dos o más variables están relacionadas.

El enfoque de la investigaciòn es cuantitativa, dado que según Gómez (2006) la perspectiva cuantitativa, la recolección de datos es equivalente a medir, en este caso se tuvo en cuenta instrumento de mediciòn dirigido a gerentes o dueños de empresas prestadoras de servicio de restaurante del Municipio de Florencia, instrumento que contó con variables medibles, porque se basó en la recopilación de normas e información a través del trabajo de campo, con el objetivo de caracterizar y conocer grupos homogéneos de fenómenos, que permiten evidenciar sistemáticamente comportamientos de hechos a partir de criterios teóricos, 0 cualquier otro fenómeno que sea sometido a un análisis, que para el caso consiste no solo en analizar aspectos teóricos, si no en describir un fenómeno que puede afectar social y económicamente a un grupo determinado de contribuyentes que deben cumplir unas obligaciones tributarias.
La investigación desarrollada fue de campo dado que la fuente principal de información, fueron los prestadores del servicio de restaurante, cuya información fue depurada por una variedad de prestadores, atendiendo a la definición de restaurante otorgada por la Ley 1607 del 2012 a través del artículo 512-8 del Estatuto Tributario.

La investigación se llevó a cabo en tres etapas, la primera comprendió actividades relacionadas con recolección de la información necesaria: base de datos de los establecimientos inscritos como restaurantes ante la cámara de comercio en el 2014, revisión documental de la normatividad existente sobre aspectos importantes de la Reforma Tributaria, la aplicación de un cuestionario a una muestra que corresponde al $28 \%$ del total de empresarios propietarios de los establecimientos catalogados como restaurantes y que se encuentran en el régimen simplificado del impuesto nacional del consumo; la segunda consistió en el análisis de la información recolectada mediante la aplicación del instrumento de investigación anteriormente descrito, que permitieron determinar el cumplimiento de las obligaciones tributarias de estos responsables, y la tercera etapa fue la elaboración del informe final con los resultados obtenidos.

El análisis estadístico de la información colectada fue desarrollado con el paquete SPSS v.14 (Norusis, 1990; Lizasoain y Joaristi, 2003) Cubre tanto las necesidades elementales para la obtención de estadísticas descriptivas y gráficos para el análisis exploratorio, como métodos avanzados de modelación estadística y análisis multivariado. Una de sus fortalezas es la sencillez de su interfaz combinada con capacidades profesionales para el cálculo y el manejo de datos. 


\section{RESULTADOS Y DISCUSIÓN:}

Con la caracterización de los establecimientos registrados como restaurantes en el municipio de Florencia, se pudo establecer el tipo de establecimiento y clase de servicio que se ofrecen en cada una de las empresas encuestadas.

\section{Figura $\mathrm{N}^{\circ} 1$}

Tipo de establecimiento versus Tipo de servicio prestado.

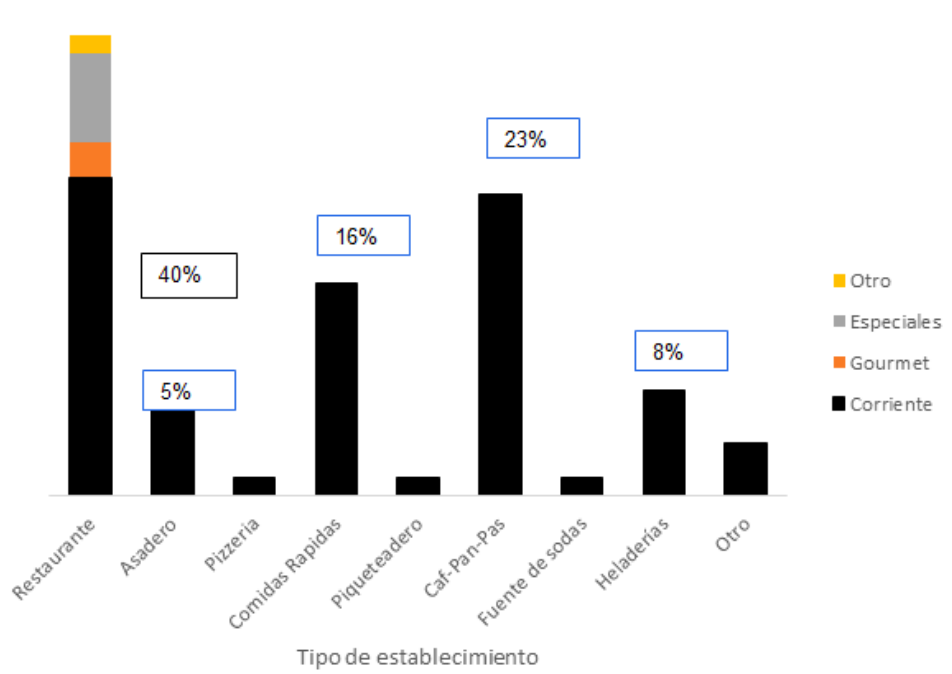

Fuente: Elaboración Propia

De las 104 empresas encuestadas, con relación al tipo de establecimiento, los más representativos son los restaurantes con el 40\%, seguido de las cafeterías, panaderías o pastelerías (Caf-Pan-Pas) con un $23 \%$, y posteriormente se encuentran los que ofrecen comidas rápidas con un $16 \%$, con una menor representación están las heladerías con un $8 \%$ y los asaderos $5 \%$ y finalmente con una baja representación se encuentran las pizzerías con un $2 \%$ y los piqueteaderos, las fruterías y fuentes de soda con el $1 \%$ para cada uno de estos tipos. (Figura 1.)
En seis de las empresas encuestadas, se seleccionaron más de un tipo de establecimiento, además de restaurante escogieron asadero, comida rápida o piqueteadero, y teniendo en cuenta que el término "restaurante" comprender un servicio más amplio o por lo menos con más variedad de productos, estos casos se reportan como restaurante.

De acuerdo al tipo de servicio que prestan los establecimientos, el más representativo es la comida corriente ofrecido por 92 empresas (83\%), seguida de la comida gourmet y de platos especiales. Hay solamente cinco empresas que prestan más de un tipo de servicios, combinando la comida corriente, con gourmet, bufet y/o platos especiales.

Figura 2.

Tiempo de prestación de servicio de las pymes del sector restaurante en Florencia Caquetá.

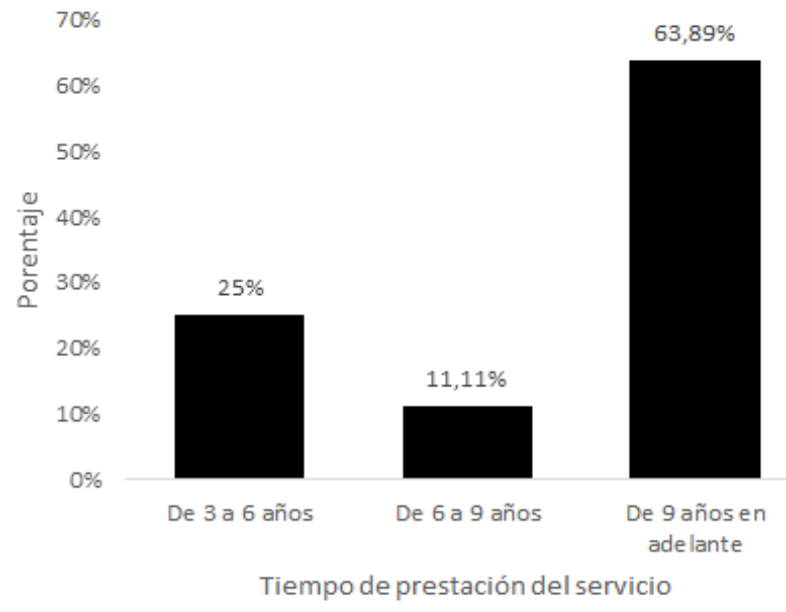

Fuente: Elaboración Propia 
ISSN: 1794-9920 Julio - Diciembre de 2018

Volumen 18 Número 2, Año 2018 Págs. 67 - 77
Tabla $N^{\circ} 1$.

Establecimientos que de acuerdo a la denominación, actividad o CIIU se categorizan como Restaurantes

\begin{tabular}{lcl}
\hline DENOMINACIÓN & CANTIDAD & OBSERVACIÓN/LA DENOMINACIÓN CONTIENE LA PALABRA \\
\hline RESTAURANTE & 35 & La denominación contiene la palabra restaurante, al inicio o dentro del texto \\
& 12 & La denominación inicia con la palabra Asadero \\
\hline ASADERO & 3 & La denominación inicia con la palabra Piqueteadero \\
\hline PIQUETEADERO & 30 & La denominación inicia con la palabra Cafetería \\
\hline CAFETERIA & 25 & La denominación inicia con la palabra Panadería \\
\hline PANADERIA & 11 & Panificadora \\
\hline PANIFICADORA & 3 & Pastelería, pasteles \\
\hline PASTELERÍA & 30 & Helado, paletas o postres
\end{tabular}

HELADERÍA

Denominación que no contienen la palabra Helado, pero son establecimientos reconocidos

COMIDAS

RÁPIDAS 16 Denominación con pizzería, hamburguesa, pinchos, sandwis, CHORIZOS

AREPAS $\quad 9 \quad$ La denominación contiene la palabra arepa

\begin{tabular}{lll}
\hline CAFÉ... & 5 & La denominación contiene la palabra café
\end{tabular}

VARIEDADES 106 , La denominación con palabras como tamales, sazón, delicias, ricuras, etc.
Fuente: Camara de Comercio de Florencia, a corte junio de 2015 .

El estudio redhtetab arroja que2de los establecimientos tipo restaurantes del régimen simplificado y responsables de

Dado que los establecimientos comerciales tienen la obligación de realizar la inscripción en el registro mercantil,

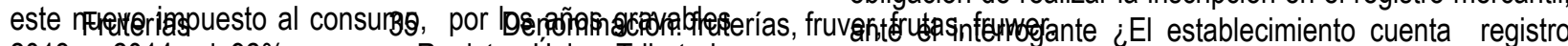
2013 y 2014, el 92\% poseen Registro Único Tributario mercantil?, los encuestados respondieron lo siguientes:

(RUT) Hoel, $1 \%$ manifiesta no tenerlo. Hoteles que además de alojamiento ofrecen servicio de restaurante

OTROS

ESTABLECIMIENT

OS

11 Sitios ubicados en zona rural o lugares alejados del epicentro de la ciudad

BALNEARIOS $\quad 19$ Denominación: parador, granja, balneario,

OTRAS

DENOMINACION

72 ES

11 La denominación Elbas, bar, centro de servicios, eventos especiales, banquetes

TOTAL 
El $85 \%$ de los establecimientos cuentan con registro mercantil; entre tanto el $14 \%$ no poseen registro mercantil, y la diferencia $(1 \%)$ no sabe o no respondieron. Igualmente se evidencia que el $80 \%$ de los establecimientos encuestados renovó oportunamente el registro mercantil en los años 2013 y 2014, y lo realizaron en forma oportuna, el $20 \%$ restante se distribuye entre la no renovación oportuna $(12 \%)$ y quienes no respondieron o no sabían (8\%).

El $66 \%$ de los establecimientos llevan contabilidad y el resto $34 \%$ expresaron que no. De los establecimientos que llevan contabilidad el $41 \%$ la llevan de manera sistematizada, el $5,56 \%$ lo realiza de forma manual y sólo un 12,50 utiliza la herramienta Excell, otro porcentaje mínimo manifestaron adelantarla tanto de manera sistematizada como manual.
A pesar de que no todas las empresas de este sector llevan contabilidad, las que si lo hacen que representan el $73 \%$, el $41 \%$ lo hace a través de Software, mientras que un $40,2 \%$ lo hace de forma manual y tan solo un $12,5 \%$ a través de la herramienta de Excell, esto puede presentarse dado que la mayoría de estas empresas son antiguas y son direccionadas por personas con experiencia y con conocimiento frente al funcionamiento y organización de las mismas. Figura 3.

A pesar de que no todas las empresas de este sector llevan contabilidad, las que si lo hacen que representan el $73 \%$, el $41 \%$ lo hace a través de Software, mientras que un $40,2 \%$ lo hace de forma manual y tan solo un $12,5 \%$ a través de la herramienta de Excell, esto puede presentarse dado que la mayoría de estas empresas son antiguas y son direccionadas por personas con experiencia y con conocimiento frente al funcionamiento y organización de las mismas. Figura 3.

Figura 3.Forma de generar los Estados Financieros, sector restaurantes del

\section{Municipio de Florencia Caquetá}

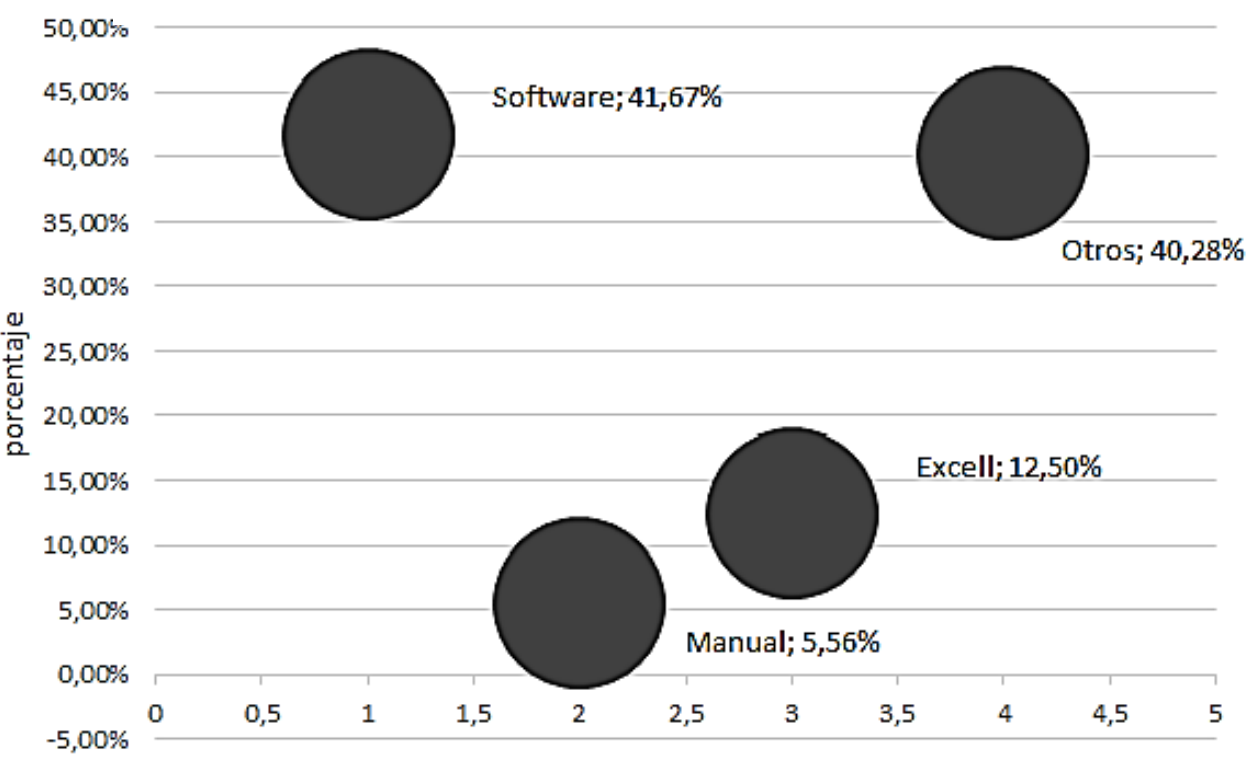

Fuente: Elaboración propia 
Figura 4. Conocimiento de la responsabilidad del impuesto al consumo, sector restaurante Mipymes de Florencia Caquetá
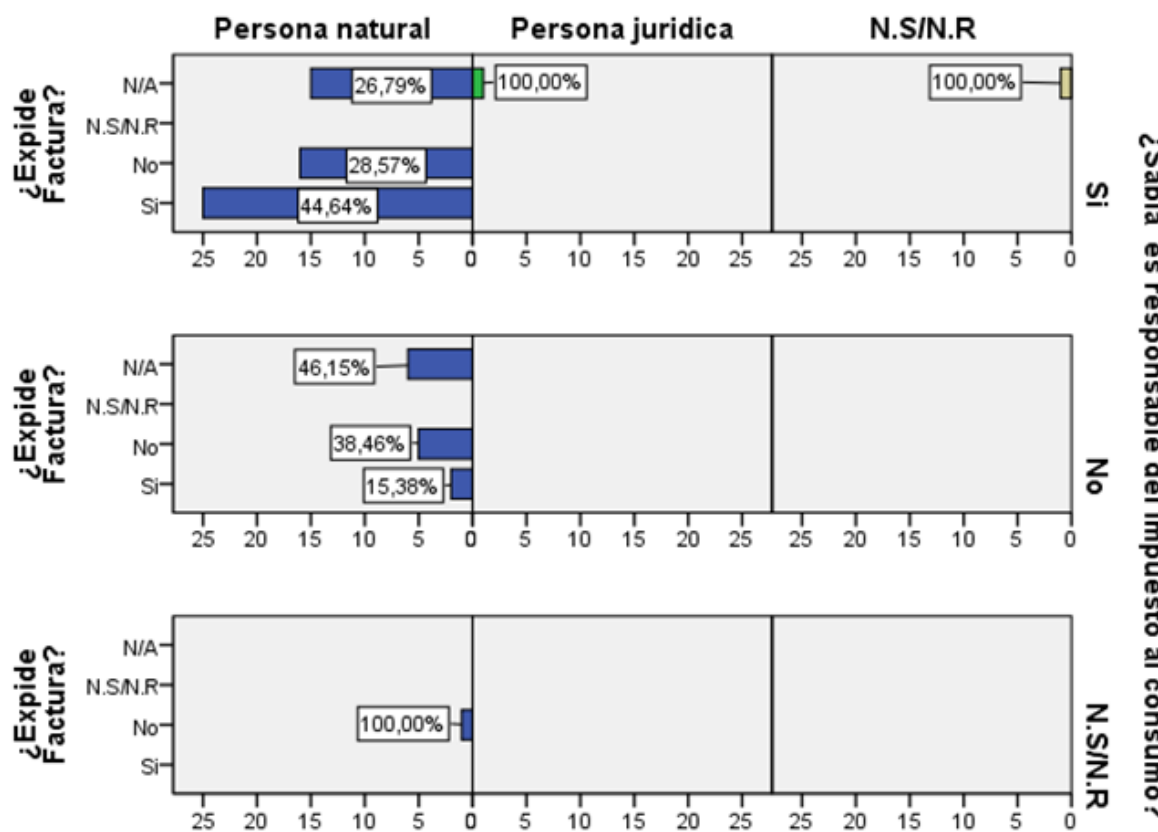

Las personas naturales que representan estas empresas, en su gran mayoría conocen y saben que son responsables del impuesto al consumo de los cuales el $44,64 \%$ expide factura. De las personas naturales que no saben que son responsables de este impuesto, sólo el $15,38 \%$ expiden factura, mientras que el $38,46 \%$ no lo hacen. Esta situación nos permite deducir que existe desconocimiento de esta norma.

Con relación a los registrados en el Régimen Simplificado el $49 \%$ expresan que expiden facturas.

Figura 5 . Régimen simplificado régimen común N/S NR pertenecen los establecimientos encuestados

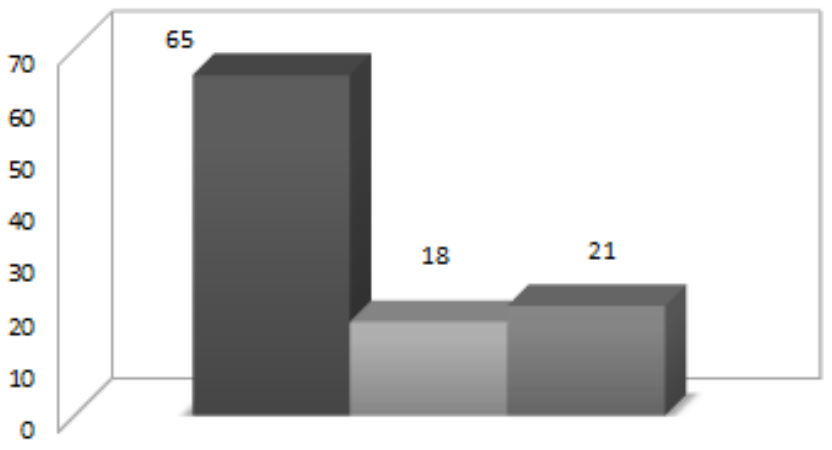

Del total de establecimientos encuestados, el $65 \%$ pertenecen al Régimen Simplificado, el $17 \%$ al Régimen Común, y el restante $21 \%$ no sabían o no respondieron al interrogante; como lo refleja la figura 5.

Una vez se conoce a qué régimen del impuesto al consumo pertenecen los establecimientos en los cuales se aplicó la encuesta, se realiza una clasificación que jermitiera saber si conoce el trámite para la presentación de la declaración "anual o bimestral" según el caso.

Es claro por ejemplo que esta pregunta aplica para los establecimientos que son responsables del impuesto al consumo que se encuentran en el Régimen Simplificado, por ello se evidencia en la pregunta la expresión "declaración anual". De las 65 empresas encuestadas, el $78 \%$ manifestaron tener conocimiento sobre el trámite para elaborar y luego realizar la presentación de la declaración anual simplificada de este impuesto y el $22 \%$ expresaron no conocer el trámite respectivo a seguir. 
Los establecimientos que manifestaron pertenecer al Régimen Común, el $100 \%$, poseen conocimiento sobre el trámite y presentación de la declaración, de las cuales el $29 \%$ realizan la declaración sin la ayuda de un Contador Público, es decir el propietario; el $60 \%$ la hacen a través de un contador y el restante $11 \%$ no saben o no dieron respuesta al interrogante.

Por otro lado los establecimientos del Régimen Simplificado que se están a paz y salvo en el pago del impuesto: el 74\% en el año 2013 y el 75\% en el año 2014; el $15 \%$ no se encontraban al día para las dos vigencias (2013-2014); y un 11\% no sabían.

Referente a las empresas registradas en el Régimen Común, se reportan los mismos resultados para la vigencia del 2013 y del 2014 , el $78 \%$ se encuentran al día con la presentación de la declaración bimestral del Impuesto al Consumo y el $22 \%$ no respondieron.

De los 104 establecimientos encuestados, el 73\% conoce sobre la responsabilidad del impuesto al consumo por ejercer la actividad de expendio de comida y bebidas a partir del 01 de enero de 2013, el 20\% no tenían conocimiento y el $7 \%$ no sabían o no respondieron al interrogante

Los establecimiento en los que se tiene conocimiento de la responsabilidad frente al impuesto al Consumo, el 96\% manifiesta estar al tanto sobre la aplicación del impuesto y el marco normativo que lo rige, y el $4 \%$ expresaron desconocerlo, a través de responder NO al cuestionamiento.

Con un porcentaje tan alto de respuesta positiva ante el conocimiento sobre la responsabilidad, la normatividad y la aplicación del impuesto al consumo, es posible indicar que para los propietarios 0 administradores de los establecimientos que prestan el servicio de expendio de comidas y bebidas preparadas, no se considera como dificultad el desconocimiento o falta de información sobre el impuesto al consumo.
Teniendo en cuenta todos los esfuerzos que se han realizado en Colombia a través de la Dirección de Impuestos y Aduanas -DIAN-, para generar los espacios virtuales, que permitan la presentación de las obligaciones fiscales por vía electrónica, obtención de información y la actualización del Registro Único Tributario, entre otros, con el propósito de brindar mayor comodidad, eficacia y por supuesto mayor eficiencia en los trámites relacionados con la parte impositiva a nivel nacional; en el presente estudio se encontró que indistintamente al Régimen al cual pertenece el establecimiento, se prefiere acudir de manera presencial ante la DIAN, para que atiendan las inquietudes que se les presenta con relación al Impuesto al Consumo.

En los establecimientos que pertenecen al Régimen Simplificado, un $82 \%$ empresas prefieren presentarse de manera presencial ante la DIAN para que les resuelvan sus inquietudes con relación al impuesto de Consumo; y un $15 \%$ no respondieron al cuestionamiento.

Con relación a las empresas que se encuentran clasificadas en el Régimen Común con relación al Impuesto al Consumo, el $89 \%$ expresan su preferencia para acudir personalmente a que le resuelvan las inquietudes frente al Impuesto objeto de estudio; sólo dos establecimientos manifestaron acudir a medios diferentes como escrito y correo electrónico, en una representación porcentual igual para cada uno $(5,5 \%)$.

Estos resultados permite deducir que los administradores de los establecimientos comerciales que prestan el servicio de venta de comidas y bebidas y líquidos preparadas no utilizan la plataforma de la DIAN para solicitar asesoría 0 realizar las consultas frente al Impuesto al Consumo, por tanto la plataforma tecnológica de la DIAN, no constituye una dificultad para el cumplimiento de la normatividad relacionada con el Impuesto objeto de estudio.

Al revisar la norma y frente a lo que hemos verificado, gran parte de los propietarios no aplican el impuesto nacional al consumo en el momento de prestar el servicio, podría presentarse situaciones que por su definición contemplada en la reforma "los establecimientos, que adicionalmente a otras actividades comerciales presten el servicio de 


\section{CONCLUSIONES:}

inciso" 5. Indicando con ello que todo establecimiento que no necesariamente sea de comercio, pues no hace distinción en este sentido y que realice otro tipo de actividades comerciales, distintas a las de expender comidas pero también presten el servicio de expendio de comidas, se considerará que presta un servicio de restaurante y por tanto es responsable del impuesto al consumo. En la práctica significa que si una persona tiene distintas actividades comerciales y entre ellas la de expender comidas bajo el concepto de servirlas para comer en el sitio o para llevarlo por cuenta del cliente o llevarlo por el servicio a domicilio, pues será sujeto pasivo de este nuevo impuesto.

Pero como lo plantea López (2013) se presentan casos en donde ha sido bastante complejo definir su ubicación en una de las definiciones anteriores, es así que La industria láctea, para comercializar sus productos, lo hace a través de distintos canales y uno de ellos son los puntos de venta en donde se comercializan productos lácteos y derivados (comidas y bebidas); es decir, leche (exenta), quesos maduros y frescos, yogures y productos que se conocen como fermentados (kumis, avenas, etc), además también venden ensaladas de frutas, miel con cuajada, fresas con crema y otros como pastelería, como galletas, tortas de queso, heladería.

Los clientes se acercan a comprar la leche (exenta) y quesos (algunos gravados) para llevar a la casa, pero mientras esperan a ser atendidos, pueden tomar kumis con tortas y sentarse; además, el punto de venta también presta el servicio de despachos a domicilio; es decir, se dan las tres formas que contiene en numeral 3 del Artículo 71 de la Reforma Tributaria.

Como se aprecia, podría pensarse que se está frente al concepto de restaurante, esto es confuso porque allí no se vende con exclusividad esos alimentos, pero también hay exentos y para determinar la base gravable para impuesto al consumo no se tiene en cuenta "los bienes excluidos", sin embargo, la norma no dice nada sobre los exentos. ¿Cómo se debe actuar en estos casos?. El Decreto reglamentario intenta dar luces pero no lo logra.

Muy de acuerdo con el análisis realizado por López \& Diaz, "De las definiciones anteriores y su análisis se podría concluir que todos los expendedores de comidas son responsables del impuesto al consumo y al igual aquellos que de manera exclusiva se dediquen a proveer a estos expendedores pero se hicieron excepciones.
Teniendo en cuenta que la definición de restaurante establecida por estatuto tributario según el artículo 512-8 es amplia en donde se encuentran expresiones como "independientemente de la denominación que se le dé al establecimiento..." y "...los establecimientos, que adicionalmente a otras actividades comerciales presten el servicio de comidas...", se requiere de una información completa frente a la identificación puntual de la actividad a la que se dedican los establecimientos de comercio dado que la sola razón social no garantiza obtener el número real de establecimientos existentes en Florencia que estarían categorizados como restaurantes. (El subrayado es propio).

El trabajo de campo realizado a las empresas objeto de estudio correspondiente a los años gravables 2013 y 2014 , se aplicó en los primeros meses del años 2016 tomando para ello la normatividad vigente con referencia a la ley 1607 del 2012; dado que a la fecha de recopilar la información para la realización del presente estudio no había sido expedida la reforma tributaria mediante ley 1819 del 2016, la cual trajo consigo modificaciones al régimen simplificado del Impuesto Nacional al Consumo en lo que tiene que ver con el servicio de restaurantes y otros aspectos.

Con relación al tiempo de prestación del servicio de restaurante en la ciudad, fue posible establecer que muchos de los establecimientos funcionaron por varios años en la ilegalidad, debido a que al cotejar la fecha suministrada en la encuesta con la reportada en la información que posee la Cámara de Comercio, se presentaron diferencias con el tiempo de servicio.

Dentro de la formalización, se encuentran el Registro Mercantil ante Cámara de Comercio y la inscripción en el Registro Único Tributario (RUT) ante la DIAN, al realizar la trazabilidad de las respuestas obtenidas, se encontró que el $92 \%$ de los establecimientos encuestados se encuentran inscritos en el RUT y el $85 \%$ poseen Registro Mercantil, es decir que se presenta una inconsistencia, si tenemos en cuenta que para que un establecimiento de comercio exista debe encontrarse registrado ante Cámara de Comercio y contar con el respectivo Registró Mercantil. 
Es posible evidenciar que en su mayoría son personas naturales, correspondientes al régimen simplificado quienes ejercen actividades enmarcadas en el concepto de restaurantes. Así mismo que dichos establecimientos se encontraban al día en la presentación de la declaración anual simplificada del Impuesto al Consumo entre el $74 \%$ y $75 \%$, correspondiente a la vigencia 2013 y 2014 , respectivamente; el $15 \%$ manifestaron no encontrarse al día, para las dos vigencias; y la representación restante no respondieron o no sabían.

Para el Régimen Común el $78 \%$ de los establecimientos se encuentran al día con la presentación de las declaraciones bimestrales del impuesto al consumo en las vigencias 2013 y 2014; y el 22\% restante, no respondieron o manifestaron no saber.

Referente a las dificultades que fueron consideraras inicialmente por los investigadores como causa para el cumplimiento con las obligaciones del impuesto al consumo, se encontraba el desconocimiento de la responsabilidad, del marco normativo y la aplicación del Impuesto al consumo, pero fue posible a través de la aplicación de la encuesta, establecer que éstas no son consideradas 0 catalogadas como dificultades 0 impedimentos para responder ante la obligación tributaria.

Otra de las dificultades para los contribuyentes, es la plataforma virtual de la DIAN, pero como resultado se obtuvo que este no es el medio preferido para realizar consultas frente al Impuesto al Consumo.

\section{REFERENCIAS:}

Congreso de la república (2002). Ley 1607 de 2012, artículos 71 al 83. Bogotá.

Congreso de la república (2014). Ley 1739 de 2014. Bogotá.

Cusguen o. E (2015) Estatuto Tributario Anotado y sus decretos reglamentarios. ISBN 978-958-769-230-3 Bogotá.

Dankhe, G L (1986), Investigación y comunicación, McGraw Hill , México.

Decreto Reglamentario 2623 (2014) Art. 30. Ministerio de Hacienda y Crédito Público de Colombia
Estatuto Tributario. Decreto 624 (1989), art. 641, Presidencia de la República de Colombia.

Gómez, M, M. (2006). Introducción a la Metodología de la Investigación Científica. Córdoba, Argentina. Edit. Brujas

Guevara M. D. H. (2014) Análisis normativo y conferencias impartidas; Líder de Investigación Contable y Tributaria. Bogotá.

Guevara M. D. H. (2014), Cartilla práctica INC, ISBN 978-9588515-31-1 Bogotá. página 6.

Hernández, S., R.;Fernández C, C.;Baptista L, P.,(1997) Metodología de la Investigación. Mc Graw Hill, México

Lerma, H. (2002) metodología de la investigación: Propuesta anteproyecto y proyecto. Pp.52-67. ECOE Ediciones. 2da Edición.

López, D, G.A., artículo de opinión: El embeleco del impuesto nacional al consumo, Actualicese, 2013, consultado en http://actualicese.com/opinion/elembeleco-del-impuesto-nacional-alconsumo-gustavo-adolfo-lopez-diaz/

Ministerio de Hacienda y Crédito Público (2013). Decreto 0803 del 24 de abril del 2013. Bogotá

Ministerio de Hacienda y Crédito Público (2014). Decreto Reglamentario 2623 de 2014. Bogotá

Norusis, M.J. (1990). Manual of SPSS/PC+.Michigan: SPSS Inc

Reforma Tributaria (2014) Ley 1739, art.51. Congreso de la República de Colombia 